\title{
Activity types of galaxies selected from HRC/BHRC sample
}

\author{
G. M. Paronyan ${ }^{1 *}$, A. M. Mickaelian ${ }^{1}$, H. V. Abrahamyan ${ }^{1}$, \\ G. A. Mikayelyan ${ }^{1}$ \\ ${ }^{1}$ NAS RA V. Ambartsumian Byurakan Astrophysical Observatory (BAO), Armenia \\ *E-mail: paronyan gurgen@yahoo.com
}

\begin{abstract}
In this study we carry out detailed spectral classification of 123 AGN candidates from the Joint HRC/BHRC sample, which is a combination of HRC (Hamburg-ROSAT Catalogue) and BHRC (ByurakanHamburg-ROSAT Catalogue). These objects were revealed as optical counterparts for ROSAT X-ray sources, however spectra for 123 of them are given in SDSS-III, IV without definite spectral classification. We studied these 123 objects using the SDSS spectra and revealed the detailed activity types for them.Three diagnostic diagrams and direct examination of the spectra were used to have more confident classification. We also made identification of these sources in other wavelength ranges and calculated some of their parameters.
\end{abstract}

Keywords: X-ray; AGN candidates; spectral classification; activity types

\section{Introduction}

ROSAT data are mainly listed in two catalogs: ROSAT Bright Source Catalogue (BSC) (Voges 1999) and ROSAT Faint Source Catalogue (FSC) (Voges 2000). They are clearly distinguished from each other by X-ray flux expressed in count-rate (CR; the number of particles registered by the receiver per unit time, namely per $1 \mathrm{sec})$.

Among the identification works, the ROSAT Bright Sources (RBS, Schwope $2000)$ is well-known. $2012 \mathrm{BSC}$ sources with $\mathrm{CR} \geq 0.20$ and $|\mathrm{b}|>30^{\circ}$ have been optically identified. However, most of the identified sources come from the Hamburg Quasar Survey (HQS, Hagen 1995), which was used as a basis for optical identifications.

Two main projects have been carried out: Hamburg-ROSAT Catalogue (HRC, Zickgraf 2003) and Byurakan-Hamburg-ROSAT Catalogue (BHRC, Mickaelian 2006). HRC is based on ROSAT-BSC and BHRC is based on 
ROSAT-FSC fainter sources (down to $\mathrm{CR}=0.04$ to have confident X-ray sources) at $|\mathrm{b}|>20^{\circ}$ and $\delta>0^{\circ}$ area.

In both catalogues, the selection of optical sources was made due to the following advantages of HQS:

1. The survey covers the entire extragalactic northern sky with $\delta>0^{\circ}$ and $|\mathrm{b}|>20^{\circ}$.

2. Spectra were received with the help of an objective prism with a dispersion $1390 \AA / \mathrm{mm}$ allowing follow spectral energy distribution (SED) and notice some broad emission and absorption lines.

3. All plates of the survey are digitized with high quality and are accessible for studies.

4. HQS allows a quick identification of objects and finding their data in other catalogues.

We combined these two Catalogues and created a new homogeneous and complete catalogue of X-ray selected AGN, which covers all the northern sky limited by high galactic latitudes $\left(\delta>0^{\circ},|\mathrm{b}|>20^{\circ}\right)$, and with $\mathrm{CR}>$ 0.04. After some checks from various available catalogs, we have excluded a number of objects and included some missed AGN and finally it contained 4253 AGN or their candidates.

Out of the 4253 HRC-BHRC objects, 3369 sources were confirmed as AGN by means of optical spectral classifications; the main criteria in VCV13 and BZCAT (Veron-Cetty 2010, Massaro 2012), 173 in Paronyan (2019) and the rest 711 are left as AGN candidates.

Out of these 711, for 123 AGN candidates in our sample there are spectra from SDSS-III and IV; we are carrying out a detailed spectral classification thus introducing new AGN or rejecting some objects.

\section{Observing material}

As observing material we had 123 spectra of HRC-BHRC objects from SDSS DR10-14 (Ahn 2014, Alam 2015, Albaretti 2017, Abolfathi 2018). Spectroscopic redshifts, intensities (assigned as "heights") and equivalent widths of spectral lines for 123 of them from SDSS DR10-DR14 are available.

Very often SDSS measurements from their spectra are based on very lowquality lines at the level of noise. These automatic measurements give some artificial numbers that indicate non-real data. So, one needs to carefully check the spectra along all wavelengths and decide which measurements should be used for further studies. Especially important are those, which 
are being used in the diagnostic diagrams (H $\beta$, [OIII] 5007 $\AA$, [OI] $6300 \AA$,

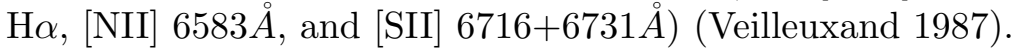

\section{Classification Principles}

We have used several methods for classification of our spectra

- By eye examination (taking into account all features and effects).

- By diagnostic diagram using $[\mathrm{OIII}] / \mathrm{H} \beta$ and $[\mathrm{OI}] / \mathrm{H} \alpha$ ratios.

- By diagnostic diagram using $[\mathrm{OIII}] / \mathrm{H} \beta$ and $[\mathrm{NII}] / \mathrm{H} \alpha$ ratios.

- By diagnostic diagram using $[\mathrm{OIII}] / \mathrm{H} \beta$ and $[\mathrm{SII}] / \mathrm{H} \alpha$ ratios.

Classification by eye has been done to compare with the classification by diagnostic diagrams and because not all objects appeared on them. Besides, the broad emission line component is not taken into account on the diagnostic diagrams, and this may be crucial for the classification of Seyfert 1.2-1.9 subclasses. Roughly, we distinguish Seyferts from LINERs by the criteria: $[\mathrm{OIII}] / \mathrm{H} \beta>4$, and AGN from $\mathrm{HII}$ by $[\mathrm{NII}] / \mathrm{H} \alpha>2 / 3,[\mathrm{OI}] / \mathrm{H} \alpha>$ 0.1 criteria.

\section{Results of Study of Spectra and Classification}

We started studying spectra with identifications of spectral lines. We have used only lines having intensities $3 \sigma$ over the noise level. $\mathrm{H} \beta$ also appears in absorption on most of these spectra. We studied the influence of $\mathrm{H} \beta$ absorption component on the emission one, which is important for using of the numerical data given in SDSS tables. After identifications of the emission lines we decided which of them should be used to build diagnostic diagrams (Veilleuxand 1987).

On diagnostic diagrams the narrow-line AGN are separated into 3 main groups (HII, Sy, LINER). In addition, there are objects in intermediate areas, which have been classified as Composites (Veilleuxand 1987) having both AGN and HII features.

\section{Summary and Conclusion}

We have created sample of X-ray selected AGN candidates and carried out spectroscopic investigation for those objects having SDSS spectra. 123 objects appear in this list and we have classified them by activity types using three diagnostic diagrams and eye examination of the spectra (to be 
complete in classification of broad line AGN). Many Seyferts, LINERs, Composites and Starburst have been revealed. We have applied all possible parameters for fine classification to distinguish between narrow and classical broad line Seyferts, and to identify all details related to Seyfert subtypes depending on the strength of their broad components. We have introduced subtypes of NLS1, namely NLS1.0, NLS1.2, NLS1,5 and NLS1.8, giving more importance to these details (Osterbrock 1980, Winkler1992, Osterbrock 1985, Heckman 1980, Ho 1997, Weedman 1977, Veron 1997). Further accumulation of statistics may provide possibilities to understand the physical differences.

These 123 objects have been cross-correlated with GALEX (Bianchi 2011), SDSS, 2MASS (Skrutskie 2006) NIR, All WISE (Cutri 2013), IRAS PSC (Beichman 1988), aFSC (Moshir 1992), NVSS (Condon 1988), and FIRST (Becker 1997) catalogues.

We have calculated all possible physical parameters of the studied objects: radial velocities, distances, absolute magnitudes, luminosity, etc.

One of the most intriguing class of objects among the X-ray sources are absorption line galaxies. The brightest ones may just appear in this sample due to their integral high luminosity, however we find that many such objects have low luminosity and still appear to be strong X-ray sources. We consider these objects as possible hidden AGN. The optical spectra do not show any signatures of emission.

\section{References}

Abazajian, K. et al. 2009, ApJS 182, 543

Abolfathi, B. et al., arXiv:1707.09322v3, (2018)

Ahn, C. P.; Alexandroff, R.; Allende Prieto, C.; Anders, F.; Anderson, S. F.; Anderton, T.; Andrews, B. H.; Aubourg, E.; et al. 2014, ApJS 211, 17

Alam, S. ; Albareti, F. D. ; Allende Prieto, C. et al., Astrophysical Journal Supplement 219 (2015) 12

Albaretti, S. D. et al., arXiv:1608.02013v2 , (2017)

Beichman, C. A.; Neugebauer, G.; Habing, H. J.; Clegg, P. E.; Chester, Thomas J. 1988, Infrared astronomical satellite (IRAS) catalogs and atlases

Becker, R. H.; Helfand, D. J.; White, R. L.; Gregg, M. D.; LaurentMuehleisen, S. A. 1997, ApJ 475, 479

Bianchi, L.; Herald, J.; Efremova, B.; Girardi, L.; Zabot, A.; Marigo, P.; Conti, A.; Shiao, B. 2011, ApSS 335, 161

Condon, J. J.; Cotton, W. D.; Greisen, E. W.; Yin, Q. F.; Perley, R. A.; Taylor, G. B.; Broderick, J. J. 1998, AJ 115, 1693

Cutri, R. M., et al. 2013, IPAC/Caltech 
Hagen, H.-J.; Groote, D.; Engels, D.; Reimers, D. 1995, A\&AS 111, 195 Heckman, T.M. 1980, A\&Ap 87, 152

Ho, L. C. ; Filippenko, A. V. ; Sargent, W. L. W., 1997, Proceedings of IAU Colloquium No. 159

Massaro, E.; Giommi, P.; Leto, C.; Marchegiani, P.; Maselli, A.; Perri, M.; Piranomonte, S.; Sclavi, S. 2012, A\&A 495, 691 (2009), online version 2012

Mickaelian, A. M.; Hovhannisyan, L. R.; Engels, D.; Hagen, H.-J.; Voges, W. 2006, A\&A 449, 425

Moshir, M.; Kopan, G.; Conrow, T.; Hacking, P.; Gregorich, D.; Rohrbach, G.; Melnyk, M.; et al. 1992, IRAS Faint Source Survey, Version 2, JPL D-10015 8/92, (IPAC)

Osterbrock, D. E.; Pogge, R. W., 1985, ApJvol. 297, p. 166-176

Osterbrock, D.E., 1980, Proc. Texas Symposium on Relativistic Astrophysics, 9th, Munich, West Germany, Dec 14-19, 1978, New York, New York Academy of Sciences, p. 22

Paronyan, G. M. et al. 2019, in press

Schwope, A.; Hasinger, G.; Lehmann, I.; et al. 2000, AN 321, 1

Skrutskie, M. F.; Cutri, R. M.; Stiening, R.; Weinberg, M. D.; Schneider, S.; Carpenter, J. M.; Beichman, C.; et al. 2006, AJ 131, 1163

Veilleux, S. and Osterbrock, D. E. 1987, ApJS 63, 295

Veron, P.; Goncalves, A. C.; Veron-Cetty-P., M., 1997, A\&Ap 319, 52

Veron-Cetty, M. P.; Veron, P. 2010, A\&A 518, A10

Voges, W.; Aschenbach, B.; Boller, Th.; Brauninger, H.; Briel, U.; Burkert, W.; Dennerl, K.; et al. 2000, IAU Circ. 7432R

Voges, W.; Aschenbach, B.; Boller, Th.; Brauninger, H.; Briel, U.; Burkert, W.; Dennerl, K.; et al. 1999, A\&A 349, 389

Weedman, D.W. 1977, VistasinAstronomy 21, 55

Winkler, H., 1992, MNRAS, vol. 257, no. 4, p. 677-688

Zickgraf, F.-J.; Engels, D.; Hagen, H.-J.; Reimers, D.; Voges, W. 2003, A\&A 406, 535 